Delignières, D., Almurad, Z.M.H., Roume, C. \& Marmelat, V. (2016). Multifractal signatures of complexity matching. Experimental Brain Research, 243(10), 2773-2785. DOI: 10.1007/s00221-016-4679-4

\title{
Multifractal signatures of complexity matching
}

\author{
Didier Delignières ${ }^{1}$, Zainy M.H. Almurad ${ }^{1,2}$, Clément Roume ${ }^{1}$ \& Vivien Marmelat ${ }^{1,3}$ \\ 1. EA 2991 Euromov, University of Montpellier, France \\ 2. Faculty of Physical Education, University of Mossul, Irak \\ 3. Center for Research in Human Movement Variability, University of Nebraska at \\ Omaha, USA
}

\begin{abstract}
:
The complexity matching effect supposes that synchronization between complex systems could emerge from multiple interactions across multiple scales, and has been hypothesized to underlie a number of daily-life situations. Complexity matching suggests that coupled systems tend to share similar scaling properties, and this phenomenon is revealed by a statistical matching between the scaling exponents that characterize the respective behaviors of both systems. However, some recent papers suggested that this statistical matching could originate from local adjustments or corrections, rather than from a genuine complexity matching between systems. In the present paper we propose an analysis method based on correlation between multifractal spectra, considering different ranges of time scales. We analyze several datasets collected in various situations (bimanual coordination, interpersonal coordination, walking in synchrony with a fractal metronome). Our results show that this method is able to distinguish between situations underlain by genuine statistical matching, and situations where statistical matching results from local adjustments.
\end{abstract}

Key-words: Synchronization, coordination, complexity matching, multifractals

Corresponding author:

Prof Didier Delignières

EA 2991, Euromov, University of Montpellier

700 avenue du Pic Saint Loup

34090 Montpellier, France

didier.delignieres@umontpellier.fr

Tel: +33411759005

Fax: +33411759024 


\section{Introduction}

The concept of complexity matching (West et al. 2008) states that the exchange of information between two complex networks is maximized when their complexities are similar. This particular property requires both networks to generate $1 / f$ fluctuations, and has been interpreted as a kind of " $1 / f$ resonance" between networks (Aquino et al. 2011). In such a situation, a complex network responds to a stimulation by another as a function of the matching of their measures of complexity, i.e. the matching of their $1 / f$ fluctuations. In contrast, the response of a complex network to a harmonic stimulus is very weak as compared with that obtained with another network of similar complexity (Aquino et al. 2010; Mafahim et al. 2015).

A direct conjecture exploiting the complexity matching effect is when two complex systems become coupled, they should attune their complexities in order to optimize information exchange. This conjecture has been initially tested by Stephen et al. (2008), in a task where participants had to synchronize finger taps with a chaotic metronome. Results showed that despite the unpredictable nature of the stimuli participants where roughly able to synchronize with the chaotic metronome, with a mix of reaction and proaction. As expected the authors observed a close matching between the scaling properties of the inter-beat interval series of chaotic signals and those of the corresponding inter-tap interval series of participants.

Marmelat and Delignières (2012) evidenced similar results in an inter-personal coordination task where participants oscillated pendulums in synchrony. Results revealed low local correlations between the series of oscillation periods produced by the two participants of each dyad. The authors analyzed the scaling properties of the series of periods produced by participants, and evidenced a very close correlation between fractal exponents. Similar results were evidenced by Abney, Paxton, Dale, and Kello (2014), in the analysis of speech signals during dyadic conversations.

However, the idea that the matching of scaling exponents could be considered an unambiguous signature of complexity matching remains questionable, and it could be necessary to distinguish between the statistical matching (i.e., the convergence of scaling exponents) and the genuine complexity matching effect (i.e., the attunement of complexities). For example Fine et al. (2015), in an experiment on rhythmic interpersonal coordination, observed as in previous experiments a typical matching of scaling exponents, but suggested that this statistical matching could just result from local phase adjustments, and not from a global attunement of complexities. Delignières and Marmelat (2014) analyzed series of stride durations produced by participants attempting to walk in synchrony with a fractal metronome. They tried to simulate their empirical results by means of a model based on local corrections of asynchronies, and showed that this model was able to adequately reproduce the statistical matching observed in experimental series. The authors concluded that walking in synchrony with a fractal metronome could essentially involve short-term correction processes, and that the close correlation observed between scaling exponents could in such a case just represent the consequence of local correction processes. Torre et al. (2013) also supported this hypothesis in a tapping task where participants synchronized with different non-isochronous auditory metronomes. They evidenced that inter-tap intervals could be modeled based on the previous inter-beat interval of the metronome and a correction of previous asynchronies to the metronome, independently of the level of $1 / f$ fluctuations of the metronome (i.e. white noise or pink noise). 
Our goal in the present paper is to seek for statistical signatures that could unambiguously distinguish between genuine global complexity matching and local corrections or adjustments. To date, most papers that tried to evidence complexity matching effects worked on the basis of monofractal analysis. Here we propose to adopt multifractal analyses because they allow for a more detailed picture of the complexity of time series, and the tailoring of fluctuations that is typical of complexity matching could be considered as the product of multifractality (Stephen and Dixon 2011). One of the most appealing hypotheses about the origin of fractal fluctuations in the behavior of complex systems refers to the idea that the interactions between system's networks are governed by multiplicative cascade dynamics (Ihlen and Vereijken 2010). Such dynamics is supposed to generate multifractal, rather than monofractal fluctuations, and indeed Ihlen and Vereijken (2010) showed that it was the case in most previous analyzed series in the literature. Stephen and Dixon (2011) consider that complexity matching should be conceived as a product of multiplicative cascade dynamics, entailing a coordination of fluctuations among multiple time scales.

While monofractal processes are characterized by long-term correlations and a single scaling exponent, in multifractal time series subsets with small and large fluctuations scale differently, and their description requires a hierarchy of scaling exponents (Podobnik and Stanley 2008). We propose to assess statistical matching through the point-by-point correlation function between the sets of scaling exponents that characterize the coordinated series.

In the present paper we used the Multifractal Detrended Fluctuation analysis (MF-DFA) introduced by Kantelhardt et al. (2002), which is an extension of the Detrended Fluctuation Analysis (DFA, Peng et al. 1993). Just as DFA, MF-DFA allows to select the range of intervals over which exponents are estimated. Usually authors considers intervals from 8 or 10 data points, in order to allow a proper assessment of statistical moments, up to $N / 4$ or $N / 2$ ( $N$ representing the length of the analyzed series), in order to get at least four or two estimates of these moments. Quite often, however, series present different scaling regimes over the short and the long term, and authors perform separate estimates over different ranges of intervals (Delignières and Marmelat 2014). Here we propose to estimate the set of multifractal exponents in first over the entire range of available intervals (i.e., from 8 to $N / 2$ ), and then over more restricted ranges, progressively excluding the shortest intervals (i.e., from 16 to $N / 2$, from 32 to $N / 2$, and then from 64 to $N / 2$ ). We expect to find in both cases (local corrections or global matching), a strong correlation pattern between exponents when considering long length intervals (i.e, 64 to $N / 2$ ). If synchronization is just based on local corrections, we consider that this close statistical matching in long intervals is just the consequence of the short-term, local coupling between the two systems. As local corrections between unpredictable systems remains approximate, we hypothesize that correlations should dramatically decrease when intervals of shorter durations are taken into consideration. In contrast, in the case of genuine complexity matching, the synchronization between systems is supposed to emerge from interactions across multiple scales. We then hypothesize to find in this case close correlations, even when considering the entire range of intervals, from the shortest to the longest. 


\section{Methods}

In the present paper we re-analyze a set of experimental series which were previously used by Delignières and Marmelat (2014), in a first attempt to derive statistical signature of complexity matching from monofractal analyses. All studies have been approved by the local ethics committee and have therefore been performed in accordance with the ethical standards laid down in the 1964 Declaration of Helsinki. All participants gave their informed consent prior to their inclusion in the study. We first briefly present the three sets of series submitted to analysis.

\section{Bimanual coordination.}

The first set of series was collected in an experiment where twelve participants performed bimanual oscillations (Torre and Delignières 2008a; Torre and Wagenmakers 2009). This kind of bimanual coordination task has been extensively studied in the dynamical systems approach to coordination, in order to evidence the emergent properties that underlie the macroscopic behavior of complex systems (Haken et al. 1985; Schöner et al. 1986). The two limbs are considered as a system of coupled oscillators, and bimanual coordination represents a nice example of close coordination between complex (sub)systems, embedded to form a global functional system. In the present context, this first set of series represents a limit case, where coordination should be achieved by complexity matching processes.

Participants were instructed to perform smooth and regular forearm oscillations holding two joysticks, synchronizing the reversal points of the motion of the joysticks (in-phase coordination). This experiment used the synchronization-continuation paradigm: during $30 \mathrm{sec}$ participants synchronized their movements with a video model, inducing an initial frequency of $1.5 \mathrm{~Hz}$ in a first condition, and $2.0 \mathrm{~Hz}$ in a second. Then the model was removed and participants had to continue following the initial tempo during 600 cycles.

For each hand, we computed the series of periods, defined as the time intervals between two successive reversal points in maximal pronation. The mean period of oscillations of the effectors was $665.24 \mathrm{~ms}(+/-63.01)$ in the $1.5 \mathrm{~Hz}$ condition, and $524.05 \mathrm{~ms}(+/-$ $66.52)$ in the $2.0 \mathrm{~Hz}$. The standard deviation of asynchronies (i.e., the time intervals between the respective pronation reversal points of the two hands) was $18.65 \mathrm{~ms}(+/-$ $5.26)$ in the $1.5 \mathrm{~Hz}$ condition and $14.45 \mathrm{~ms}(+/-2.62)$ in the $2.0 \mathrm{~Hz}$ condition, corresponding to a mean relative phase was $-6.33^{\circ}(+/-3.76)$, and $-5.27^{\circ}(+/-6.01)$, respectively.

\section{Interpersonal synchronization}

The second set of series were collected in an experiment on interpersonal coordination (Marmelat and Delignieres 2012). In contrast with the previous example, these series represent coordination between two physically independent systems that interact for achieving a common goal. Twenty-two participants were randomly paired into eleven dyads. Participants in each dyad were instructed to perform synchronized oscillations with pendulums, in the sagittal plane, following an in-phase pattern of coordination. They were instructed to oscillate at the preferred frequency of the dyad, as regularly as possible. The task was performed in three conditions, characterized by increasing levels of coupling between participants. In the weak coupling condition, audition was limited with earplugs, and participants were instructed to visually fix a target in front of them on the wall. In the normal coupling condition, visual and auditory feedbacks were fully 
available, and participants were invited to visually fix their partner's pendulum. In the strong coupling condition, participants were instructed to cross their free arms (arm-inarm), in order to add haptic information to visual and auditory feedbacks. Series of 512 oscillations were collected in each condition. For each participant, we computed the series of periods, defined as the time intervals between two successive reversal points in maximal extension.

Results showed that dyads were able to perform adequately this coordination task, with a mean relative phase of $-2.15^{\circ}(+/-8.64)$ in the low coupling condition, $-1.67^{\circ}(+/-7.50)$ in the normal coupling condition, and $-2.36^{\circ}(+/-7.15)$ in the strong coupling condition.

The mean period of oscillations of the effectors was $1035.06 \mathrm{~ms}(+/-130.59), 1018.47$ ms (+/- 126.70), and $989.35 \mathrm{~ms}(+/-51.95)$, respectively. The standard deviation of asynchronies was $46.51 \mathrm{~ms}$ (+/- 7.50), $34.78 \mathrm{~ms} \mathrm{(+/-} \mathrm{9.10)} \mathrm{and} 37.71 \mathrm{~ms}(+/-6.06)$, respectively.

\section{Walking in synchrony with a fractal metronome}

In this experiment participants had to walk in synchrony with a fractal metronome. Eleven participants were involved in this experiment. They walked on a treadmill, and had to synchronize the right heel strikes with metronome signals administered through an earphone. Metronome signals presented fractal fluctuations with a mean $\alpha$ exponent of about 0.9 , a mean value of $1135 \mathrm{~ms}$, and their standard deviation was adjusted for obtaining a coefficient of variation of $2 \%$. Series of 512 strides, defined as the time intervals between two successive right heel strikes, were collected. Results showed that participants were able to maintain synchrony with the metronome, with a mean asynchrony of about $-52.8 \mathrm{~ms}(+/-46.9)$. The mean stride duration was $1135.53 \mathrm{~ms}(+/-$ 51.95), and the standard deviation of asynchronies was $46.94 \mathrm{~ms}(+/-11.68)$.

\section{Multifractal Detrended Fluctuation analysis (MF-DFA)}

In the present paper we used the MF-DFA method, initially introduced by Kantelhardt et al (2002). Consider the series $x(i), i=1,2, \ldots, N$. In a first step the series is centered and integrated:

$$
X(k)=\sum_{i=1}^{k}\left[x(i)-\frac{1}{N} \sum_{i=1}^{N} x(i)\right]
$$

Next, the integrated series $X(k)$ is divided into $N_{n}$ non-overlapping segments of length $n$ and in each segment $s=1, \ldots, N_{n}$ the local trend is estimated and subtracted from $X(k)$.

The variance is calculated for each detrended segment:

$$
F^{2}(n, s)=\frac{1}{n} \sum_{k=(s-1) n+1}^{s n}\left[X(k)-X_{n, s}(k)\right]^{2}
$$

and then averaged over all segments to obtain $q$ th order fluctuation function

$$
F_{q}(n)=\left\{\frac{1}{N_{n}} \sum_{s=1}^{N_{n}}\left[F^{2}(n, s)\right]^{q / 2}\right\}^{1 / q}
$$

where $q$ can take any real value except zero. In the present work we used integer values for $q$, from -15 to +15 . Note that Eq. (3) cannot hold for $q=0$, because of the diverging exponent. A logarithmic averaging procedure is used for this special case: 


$$
F_{0}(n)=\exp \left(\frac{1}{2 N_{n}} \sum_{s=1}^{N_{n}} \ln \left[F^{2}(n, s)\right]\right)
$$

Repeating this calculation for all lengths $n$ provides the relationship between fluctuation function $F_{q}(n)$ and segment length $n$. If long-term correlations are present, $F_{q}(n)$ increases with $n$ according to a power law:

$$
F_{q}(n) \propto n^{h(q)}
$$

The scaling exponent $h(q)$ is obtained as the slope of the linear regression of $\log F_{q}(n)$ versus $\log n$. Note that for stationary time series, $h(2)$ is identical to the well-known Hurst exponent $H$, and therefore $h(q)$ is called the generalized Hurst exponent.

For positive values of $q$ the generalized Hurst exponent $h(q)$ describes the scaling behavior of large fluctuations, while for negative values of $q, h(q)$ describes the scaling behavior of small fluctuations. For monofractal time series $h(q)$ is independent of $q$, while for multifractal time series small and large fluctuations scale differently and $h(q)$ is a decreasing function of $q$.

The results of the MF-DFA can then be converted into the more classical multifractal formalism by simple transformations (Kantelhardt et al. 2002): first, generalized Hurst exponents $h(q)$ are related to the Renyi exponents $\tau(q)$ defined by the standard partition function-based multifractal formalism:

$$
\tau(q)=q h(q)-1
$$

For monofractal signals $\tau(q)$ is linear function of $q$, and for multifractal signals $\tau(q)$ is nonlinear function of $q$. Another way to characterize multifractal process is the singularity spectrum $f(\alpha)$ which is related to $\tau(q)$ through the Legendre transform:

$$
\begin{gathered}
\alpha(q)=\frac{d \tau(q)}{d q} \\
f(\alpha)=q \alpha-\tau(q)
\end{gathered}
$$

where $f(\alpha)$ is the fractal dimension of the support of singularities in the measure with Lipschitz-Hölder exponent $\alpha$. The singularity spectrum of monofractal signal is represented by a single point in the $f(\alpha)$ plane, whereas multifractal process yields a single humped function.

\section{The focus-based approach to multifractal analysis}

The classical MF-DFA algorithm was shown to perform as well as other multifractal methods (Oswiecimka et al. 2006; Schumann and Kantelhardt 2011). However, especially when applied on empirical series it is known to often produce the so-called "inversed" spectra, exhibiting a zig-zag shapes rather than the expected parabolic shape (Makowiec et al. 2011; Mukli et al. 2015).

Mukli et al. (2015) have recently introduced a focus-based approach which allows to avoid this pitfall. The standard method assesses the scaling exponents $h(q)$ independently for each $q$ value. This procedure can be considered adequate if an assumption on homogeneous structuring holds for the scaling function. This property however may not always be present especially in empirical signals.

Theoretically, the moment-wise scaling functions, for all $q$ values, should converge 
toward a common limit value at the coarsest scale. Indeed, substituting signal length $(N)$ to interval length $(n)$ in Eq. (3) yields:

$$
F_{q}(N)=\left\{\frac{1}{N_{N}} \sum_{s=1}^{N_{N}}\left[F^{2}(N, s)\right]^{q / 2}\right\}^{1 / q}=\left\{F^{2}(N, s)^{q / 2}\right\}^{1 / q}=F(N, s)
$$

$F(N, s)$ can then be considered the theoretical focus of the scaling functions, and Mukli et al. (2015) proposed to use this focus as a guiding reference when regressing for $h(q)$. In essence, one can iterate on $h(q)$ as the ideal multifractal with its given focus and set of associated slopes best fitting to the observed data of the scaling function. Mukli et al. (2015) showed that this method allowed to successfully avoid the occurrence of inversed spectra.

As explained in the introduction, we first applied MF-DFA considering the widest range of intervals, from 8 to $256(N / 2)$. We then replicated this analysis by progressively focusing on longer intervals: 16 to 256,32 to 256 , and 64 to 256 .

We finally computed for each $q$ value the correlation between the individual LipschitzHölder exponents characterizing the two coordinated systems, $\alpha_{1}(q)$ and $\alpha_{2}(q)$, respectively, yielding a correlation function $r(q)$. As previously explained, we expected to find in all cases a correlation function close to 1 , for all $q$ values, when only the largest intervals are considered. Increasing the range of considered intervals should have a negligible impact on $\mathrm{r}(q)$ when coordination is based on a complexity matching effect. In contrast, if coordination is based on local corrections, a decrease in $r(q)$ should be observed, as shorter and shorter intervals are considered.

\section{Results}

\section{Bimanual coordination.}

We present in Figure 1 (upper panels) the averaged multifractal spectra of the period series, considering intervals from 8 to 256 points, in the $1.5 \mathrm{~Hz}$ condition (a) and the 2.0 condition (b). The spectra of the right and the left hand are closely superimposed in both conditions. The correlation functions between the multifractal spectra are presented in bottom panels. Correlation coefficients are plotted against their corresponding $q$ values. Four correlation functions are displayed, according to the shortest interval length considered during the analysis $(8,16,32$, or 64$)$. In all cases the correlations functions displayed very high values, close to 1.0 (Figure 1, c and d). Especially in the $1.5 \mathrm{~Hz}$ condition the correlations between spectra appeared maximal, over all $q$ values and whatever the considered intervals range. Correlations appeared slightly lower (around 0.90 ), in the $2.0 \mathrm{~Hz}$ condition, for negative values of $q$, and when the entire range of intervals were considered.

\section{Interpersonal synchronization.}

We present in Figure 2 (upper panels) the averaged multifractal spectra of the period series in the low coupling (a), normal coupling (b) and strong coupling (c) conditions. As for the previous experiment, we observed a close superimposition of the two averaged spectra, and the superimposition appeared closer as coupling strength increased. The correlation functions between the multifractal spectra are presented in bottom panels (d, e and f). In all cases the correlation function displayed very high values, close to 1.0, when analysis focused to long intervals (i.e. 32 to $N / 2$ or 64 to $N / 2$ ). 

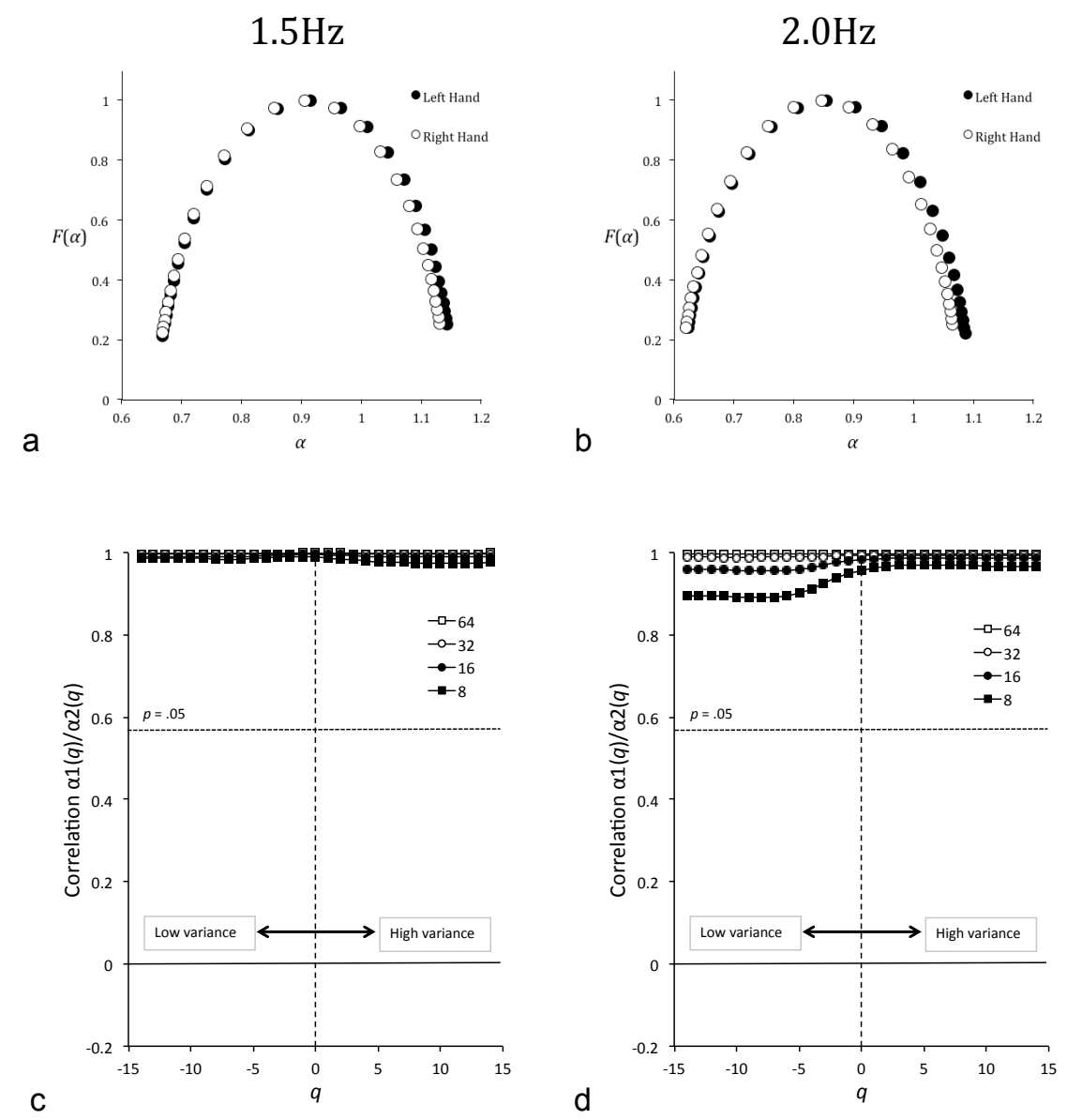

Figure 1: Bimanual coordination. Upper panels: Multifractal spectra for the right (black circles) and the left (white circle) effectors, for the $1.5 \mathrm{~Hz}$ (a) and the $2.0 \mathrm{~Hz}$ (b) conditions. Bottom panels: Correlation functions $\mathrm{r}(q)$, for the four ranges of intervals considered (8 to $N / 2,16$ to $N / 2,32$ to $N / 2$, and 64 to $N / 2$ ) , for the $1.5 \mathrm{~Hz}$ (c) and the $2.0 \mathrm{~Hz}$ (d) conditions. $q$ represents the set of orders over which the MF-DFA algorithm was applied.

\section{Walking in synchrony with a fractal metronome}

We present in Figure 3 (panel a) the averaged multifractal spectra of the stride duration series (black circles) and the metronome series (white circles). In this experiment a shift was observed between the two averaged spectra, indicating a lower level of correlation in participants series, with respect to the corresponding metronomes series. The correlation functions between the multifractal spectra are presented in the right panel (b). In contrast with the previous results, the considered range of intervals had a strong impact on the correlation function. When the smallest range was considered (64 to $N / 2$ ), the correlation function remained close to 1.0. When the range of interval was progressively enlarged, the correlation values decreased, especially for negative $q$ values corresponding to low variance epochs in the series. When all available intervals are considered (i.e. 8 to $N / 2)$, r $(q)$ presents non significant values. 

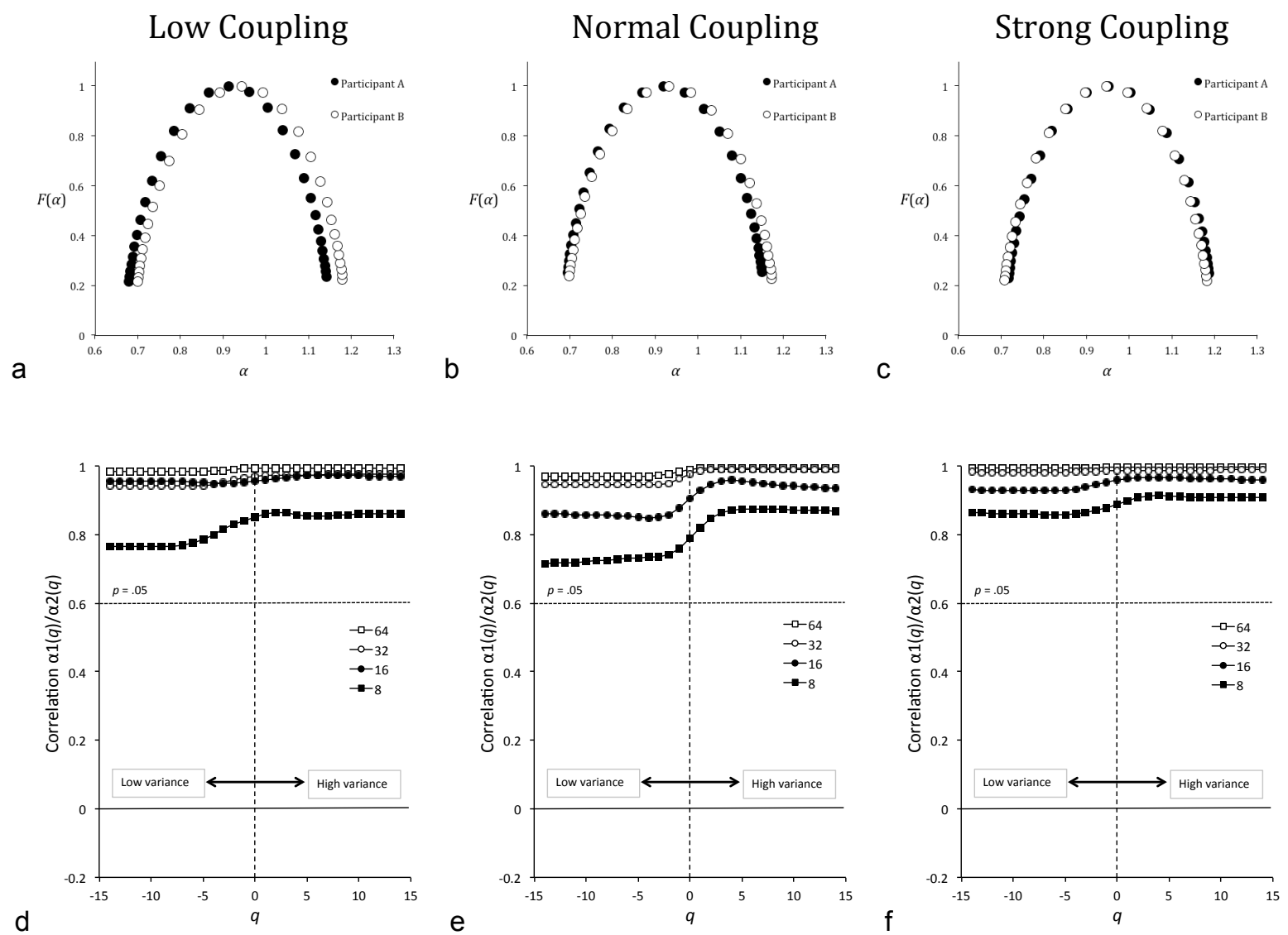

Figure 2: Interpersonal synchronization. Upper panels: Multifractal spectra for participant A (black circles) and participant B (white circle), for the low (a), normal (b) and strong (c) coupling conditions. Bottom panels: Correlation functions $r(q)$, for the four ranges of intervals considered ( 8 to $N / 2,16$ to $N / 2,32$ to $N / 2$, and 64 to $N / 2$ ), for the low (d), normal (e) and strong (f) coupling conditions.
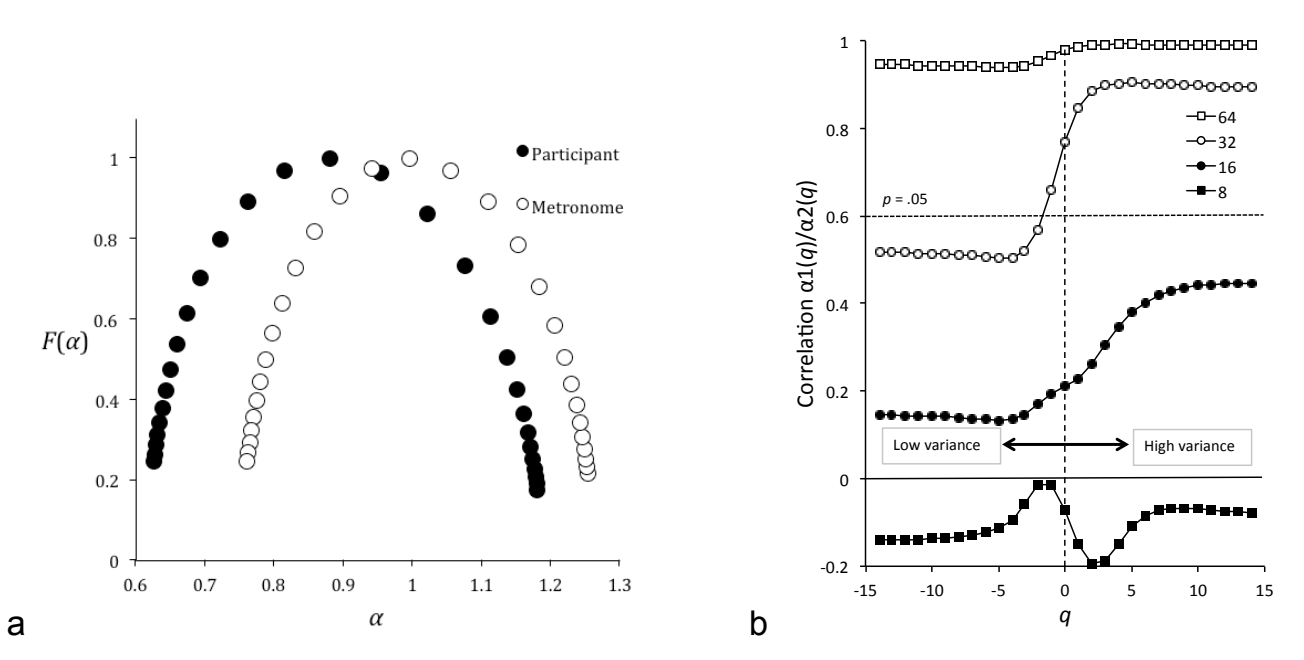

Figure 3: Walking in synchrony with a fractal metronome. Panel a: Multifractal spectra for the participant (black circles) and the metronome (white circle). Panel b: Correlation functions $\mathrm{r}(q)$, for the four ranges of intervals considered (8 to $N / 2,16$ to $N / 2,32$ to $N / 2$, and 64 to $N / 2$ ). 
In order to provide a clearer picture of the evolution of correlations with the range of considered intervals in the three experiments, we present in Figure 4 a set of scatter plots representing the relationships between the $\alpha(2)$ samples characterizing the two coordinated systems. These graphs show a global narrowing of exponent's samples, as the considered range of intervals increases. However, the decrease of correlation, especially in the third experiment, clearly arises from a weakening of the relationships between exponents.

$64-N / 2$

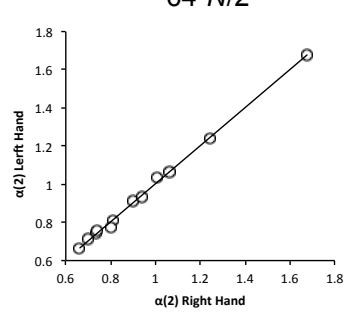

a
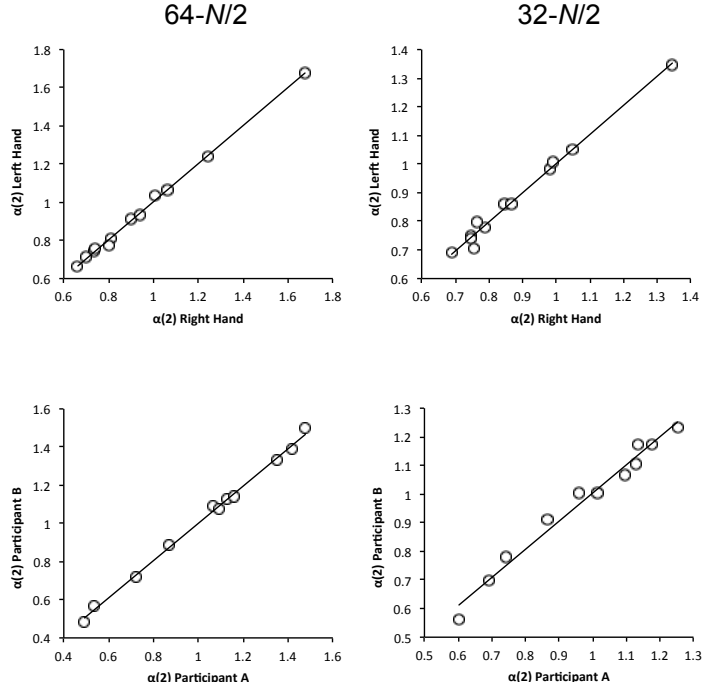

b
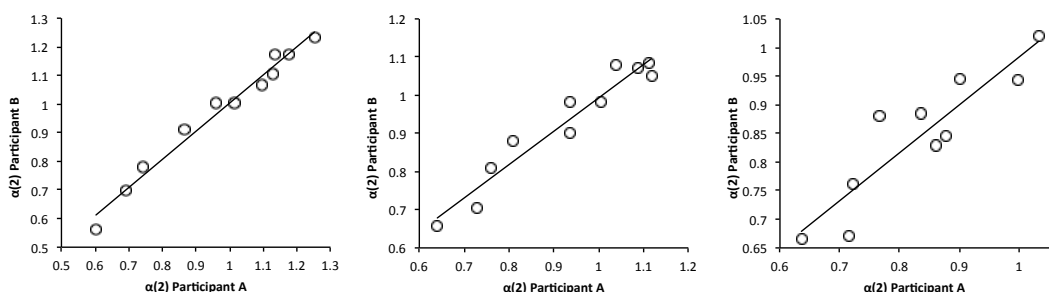

C
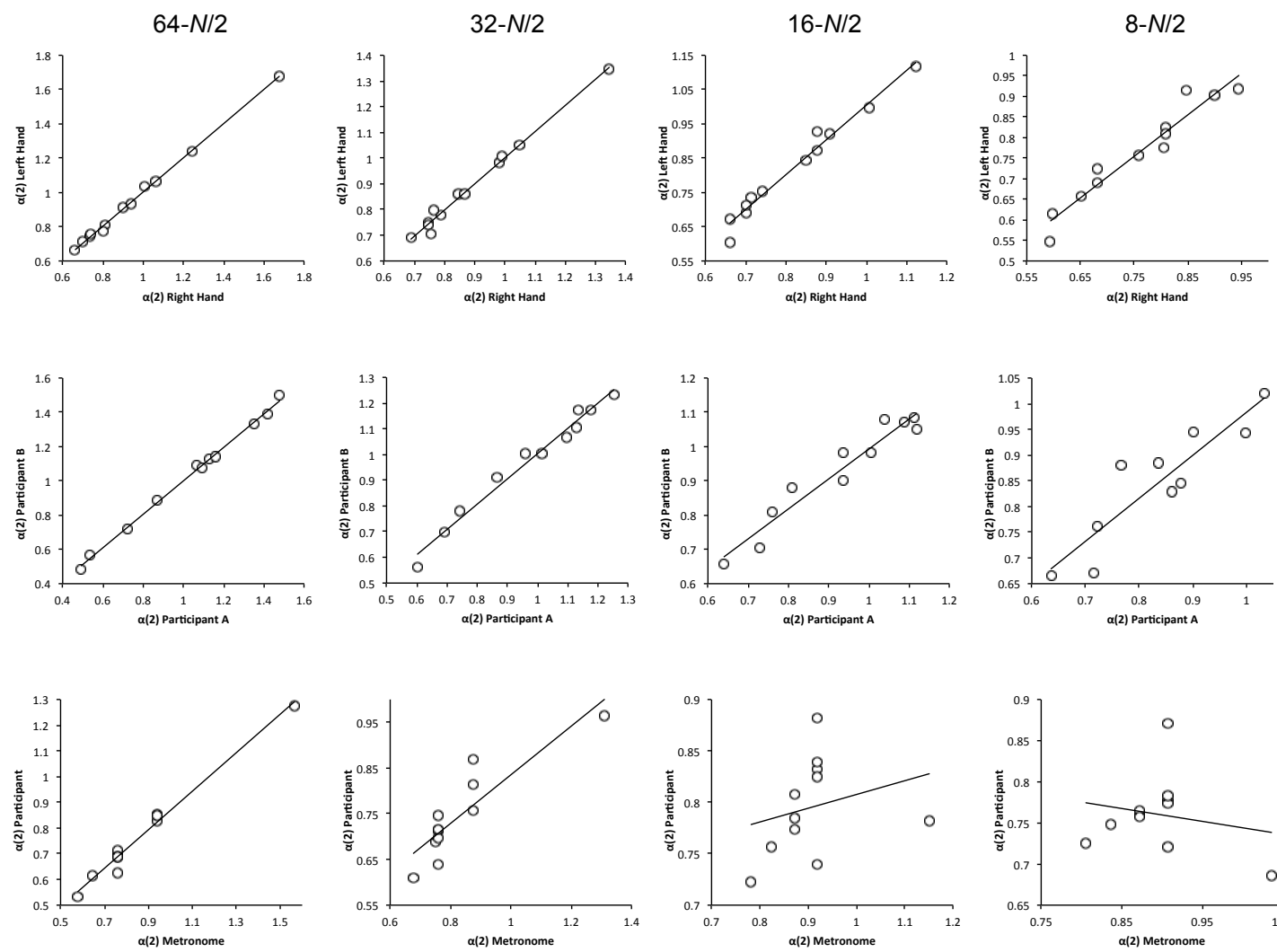

Figure 4: Scatter plots of the samples of Hölder exponents $\alpha(2)$ characterizing the coupled series, in the three experiments, for the four ranges of intervals considered (64 to $N / 2,32$ to $N / 2,16$ to $N / 2$, and 8 to $N / 2$ ). Upper row (a): bimanual coordination, F1; Middle row (b): interpersonal coordination, strong coupling; Bottom row (c): walking in synchrony with a fractal metronome.

\section{Discussion}

The present results are based on the analysis of behavioral series of relatively short length. 512 data points could be considered insufficient for deriving reliable results, especially with multifractal analyses. However, the application of time series analyses supposes that the system under study remains in stable state during the whole window of observation, and in behavioral experiments the lengthening of the task could raise problems of fatigue or lack of concentration (Delignières et al. 2005; Marmelat and Delignières 2011). On the other hand, a number of improvements have been introduced in fractal analyses, which could allow to consider with a certain confidence the results obtained from relatively short series (Delignières et al. 2006; Almurad and Delignières 2016). Such series lengths are generally considered as an acceptable compromise between the requirements of time series analyses and the limitations of hebavioral human experiments (Gilden 1997; Chen et al. 1997; Gilden 2001; Chen et al. 2001). 
We are aware that the present results should be considered with caution, and have to be confirmed by further analyses. However, the differences we observed between the three experiments are consistent with our initial hypotheses and seem sufficiently large to be regarded with some confidence.

\section{Complexity matching vs discrete local coupling}

The main result of this paper is the clear distinction between the two first experiments (bimanual coordination and interpersonal coordination) and the third one (walking with a fractal metronome). In the two first cases the correlation function revealed a clear statistical matching between multifractal spectra, whatever the range of intervals considered in the analysis. In contrast, in the third set of data the close statistical matching appeared only when the range was restricted to the lengthiest intervals (i.e., from 64 to 256), and was progressively altered when wider ranges were considered.

These results suggest that in the two first experiments synchronization was governed by a global, multiscale coordination between the two interacting systems, and in the third experiment synchronization was the result of local corrective processes. As in this experiment participants had to synchronize with a series of discrete stimuli, one could hypothesize that these correction processes work on a discrete, step-to-step basis, as suggested by Marmelat and Delignières (2012). One could argue, however, that this hypothesis should be considered with caution, as the task used in the third experiment strongly differs from those used in the others, and especially the walking task involves very large masses compared to the other tasks. Note, however, that Torre et al. (2013) proposed a similar hypothesis in an experiment where participants had to synchronize finger taps with fluctuating metronomes.

It could be interesting, for reinforcing this hypothesis, to check whether a model based on such discrete, local correction processes, could generate series yielding comparable results. We then tried to simulate series that could result from a local coupling with a fractal metronome. In this modeling study we considered that the organism produces intrinsically long-range correlated series. Indeed, a number of previous studies have shown that organisms produced long-range correlated series in self-paced conditions, and that, during synchronization with a regular metronome, this source of long-range correlation was still at work and had to be considered for properly modeling the synchronisation process (Torre and Delignières 2008b; Torre and Delignières 2009; Delignières and Marmelat 2014). We then proposed that the organism corrects the interval it intended to intrinsically produce on the basis of the previous asynchronies (Delignières and Marmelat 2014). We worked with a two-order auto-regressive model :

$$
\begin{gathered}
x(i)=y(i)-a[\operatorname{ASYN}(i-1)]-b[\operatorname{ASYN}(i-2)]+c \varepsilon(i), \\
A S Y N(i)=\operatorname{ASYN}(0)+\sum_{k=0}^{i} x(k)-\sum_{k=0}^{i} z(k)
\end{gathered}
$$

where $x(i)$ represents the series of periods effectively produced by the organism, $y(i)$ the series of virtual periods produced by the organism, and $z(i)$ the fractal metronome. $A S Y N(i)$ is the series of asynchronies between the events produced by the organism and the signals of the metronome. $y(i)$ and $z(i)$ were both modeled as fractional Gaussian noises with $H=0.9$ (mean $=1000$ and $S D=20$ ). Finally $\varepsilon(i)$ is a white noise process with zero mean and unit variance. 
This model suggests that periods are corrected on the basis of the two previous asynchronies, a hypothesis consistent with the cross-correlation functions obtained in this experiment (Delignières and Marmelat 2014).

For the present simulations we used $a=0.2, b=0.4, c=12$, and we generated 12 series of 512 data points. We present in Figure 5 (panel a) the averaged multifractal spectra for the 'participant' (black circles) and the 'metronome' (white circles). Note that these simulated results are characterized by a shift of the first spectrum, similar to that observed in experimental data (see Figure 3). Panel b represents the correlation functions $r(q)$, for the four ranges of intervals considered. As can be seen we obtained a pattern of results similar to that obtained with experimental results: when focusing on long-term intervals the correlation function remained close to one, and progressively extinguished as more and more shorter-term intervals were taken into consideration.

a

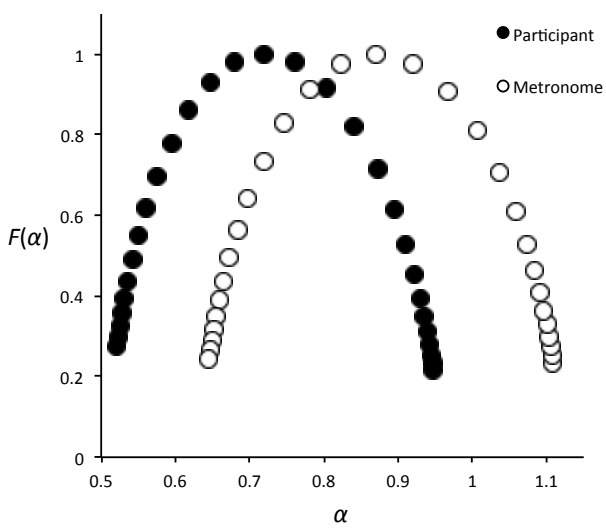

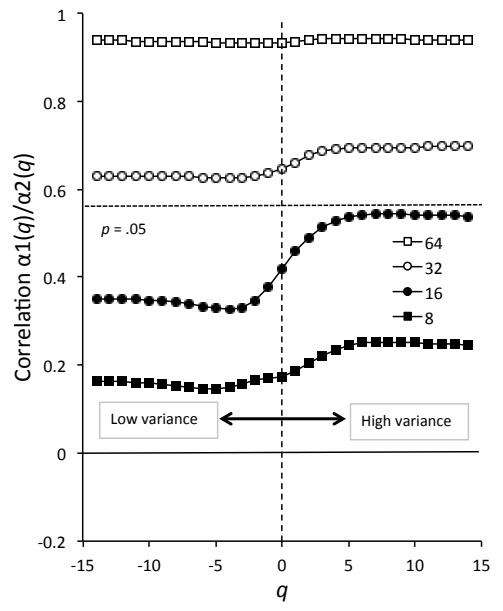

Figure 5: Simulation of the synchronization to a fractal signal by local corrections of asynchronies. Panel a: Multifractal spectra for the 'participant' (black circles) and the 'metronome' (white circle). Panel b: Correlation functions $\mathrm{r}(q)$ ), for the four ranges of intervals considered ( 8 to $N / 2,16$ to $N / 2,32$ to $N / 2$, and 64 to $N / 2$ ).

Another difference between our experiments lies in the coupling between systems: in bimanual coordination and interpersonal synchronization the systems mutually interacted but synchronization with a fractal metronome is characterized by the presence of a 'master' (the metronome) and a 'slave' (the participant). Interestingly, our results revealed an asymmetry in the alteration of correlations when larger ranges of intervals were considered: we especially observed a dramatic decrease of correlations for negative $q$-values, corresponding to low-variance epochs in the signals (Figure 3). This result was particularly obvious in the third experiment, and suggests that high variance epochs in the metronome signals allow a better synchronization, reinforcing the hypothesis of a discrete, perceptual basis of synchronization.

These results question a number of recent experiments dealing with the use of fractal metronomes with the perspective of rehabilitation purposes (Stephen et al. 2008; Hove et al. 2012; Kaipust et al. 2013; Torre et al. 2013; Rhea et al. 2014; Marmelat et al. 2014). In their seminal paper, Stephen et al. (2008) suggested that synchronization with a chaotic metronome was not based on local adjustments but rather on a global, multiscale coordination with the metronome. The present results cast doubt on this conclusion (see also Delignières \& Marmelat, 2014; Torre et al., 2013). Fractal metronomes have recently sparked a great interest, suggesting that they could mimic 
natural variability, and be used for conceiving artificial devices for training and rehabilitation, based on the complexity matching effect. Mimicking natural variability, especially with discrete signal series, seems not sufficient to generate the global and multiscale coordination hypothesized in complexity matching.

\section{Complexity matching vs continuous local coupling}

Our results, however, do not prove that the strong statistical matching observed in bimanual coordination and interpersonal coordination is due to complexity matching, as defined in the introduction. Recently, Fine et al (2015) questioned the global complexity matching hypothesis, and suggested that a local and continuous coupling between systems could underlain the statistical matching observed in such situations.

The dynamical systems approach to coordination promoted a phenomenological model based on a continuous coupling between oscillators (Haken et al. 1985; Schöner et al. 1986). This so-called HKB model accounts for coordination by non-linear coupling between two hybrid limit-cycle oscillators, based on the two oscillators' state variables (position and velocity):

$$
\left[\begin{array}{c}
\ddot{x}_{1}+\delta \dot{x}_{1}+\lambda \dot{x}_{1}^{3}+\gamma x_{1}^{2} \dot{x}_{1}+\omega^{2} x_{1}=\left(\dot{x}_{1}-\dot{x}_{2}\right)\left[a+b\left(x_{1}-x_{2}\right)^{2}\right] \\
\ddot{x}_{2}+\delta \dot{x}_{2}+\lambda \dot{x}_{2}{ }^{3}+\gamma x_{2}{ }^{2} \dot{x}_{2}+\omega^{2} x_{2}=\left(\dot{x}_{2}-\dot{x}_{1}\right)\left[a+b\left(x_{2}-x_{1}\right)^{2}\right]
\end{array}\right.
$$

where $x_{i}$ is the position of oscillator $i$, and the dot notation represents derivation with respect to time. The left side of the equations represents the limit cycle dynamics of each oscillator determined by a linear stiffness parameter $(\omega)$ and damping parameters $(\delta, \lambda$, and $\gamma$ ), and the right side represents the coupling function determined by parameters $a$ and $b$. This model has been proven to adequately account for most empirical features in bimanual coordination tasks, such as the differential stability of in-phase and anti-phase coordination modes, and the transition from anti-phase to in-phase coordination with the increase of oscillation frequency (Haken et al. 1985; Schöner et al. 1986).

One can indeed suppose that this kind of continuous coupling could be at the origin of the strong statistical matching observed in coordination experiments (Fine et al. 2015), but the multifractal signature proposed in this paper could be unable to distinguish between genuine complexity matching and such local and continuous coupling. A solution for disentangling these two hypotheses is to analyze the series produced by this model, and to compare them to those empirically observed.

However, in order to account for empirical features, the original HKB model should be slightly modified. Especially, it has been proven that in bimanual coordination, the series of periods produced by each effector and the series of relative phase contained longrange correlations, a property that the original HKB model was unable to generate (Torre and Delignières 2008a). The authors proposed to account for this behavior by replacing the fixed linear stiffness parameter $\omega$ in equations (12) by a two independent discrete series $\omega_{1, i}$ and $\omega_{2, i}$, exhibiting long-range correlation properties, and representing the inner frequencies of oscillators 1 and 2, respectively, at cycle $i$.

$$
\left[\begin{array}{c}
\ddot{x}_{1}+\delta \dot{x}_{1}+\lambda \dot{x}_{1}^{3}+\gamma x_{1}{ }^{2} \dot{x}_{1}+\omega_{1, i}{ }^{2} x_{1}+\sqrt{Q} \varepsilon_{1, t}=\left(\dot{x}_{1}-\dot{x}_{2}\right)\left[a+b\left(x_{1}-x_{2}\right)^{2}\right] \\
\ddot{x}_{2}+\delta \dot{x}_{2}+\lambda \dot{x}_{2}{ }^{3}+\gamma x_{2}{ }^{2} \dot{x}_{2}+\omega_{2, i}{ }^{2} x_{2}+\sqrt{Q} \varepsilon_{2, t}=\left(\dot{x}_{2}-\dot{x}_{1}\right)\left[a+b\left(x_{2}-x_{1}\right)^{2}\right]
\end{array}\right.
$$


Note that they also introduced white noise terms of strength $Q$ in the limit cycle equations, as suggested by Schöner et al. (1986). Torre and Delignières (2008a) showed that a relevant set of parameters allowed to simulate a stable in-phase coordination between the two oscillators, despite the intrinsic long-range correlated fluctuations injected in each oscillator. Their results replicated most empirical results, in terms of mean and standard deviation of relative phase, and also concerning the presence of long-range correlations in the series of relative phase.

We used this model for generating pairs of series of coordinated periods. We attempted to generate series reproducing the main features of the inter-personal coordination experiment, in the strong coupling condition. We used the same set of parameters than Torre and Delignières (2008a): $\delta=0.5, \lambda=0.02, \gamma=1.0, Q=0.4$. We used the "hopping model" (Delignières et al. 2008; Torre and Delignières 2008a) for simulating the longrange correlated series $\omega_{1, i}$ and $\omega_{2, i}$ around a mean value of $4 \pi$. For stabilizing in-phase coordination between the two systems, the coupling parameters were set to $a=12$ and $b=6$. These values were much stronger than those commonly reported in the literature (Fink et al. 2000; Assisi et al. 2005; Leise and Cohen 2007), but were necessary for obtaining a stable coordination (Delignières et al. 2008; Torre and Delignières 2008a). We simulated 12 sets of series of 512 data points.

Simulated series broadly reproduced experimental results, with a mean relative phase of $0.74^{\circ}(+/-6.93)$. The mean period of oscillations was $1001.14 \mathrm{~ms}(+/-50.07)$. The standard deviation of asynchronies was $19.34 \mathrm{~ms}(+/-3.38)$. We present in Figure 6 (a and b) the result of the multifractal analysis of these coordinated series. As can be seen, the correlation function $r(q)$ exhibited very high values, even when considering the widest range of intervals. This result appears similar to that obtained with the experimental series (Figure 2), suggesting that this kind of local, continuous coupling, could indeed underlay the observed statistical matching between series.

However, a closer look to the coupled period series casts some doubts about this conclusion. We present in Figure 6 two examples subsets of coupled series (50 points), the first graph (c) corresponding to representative experimental series, and the second (d) to simulated series. These graphs suggest that while providing comparable statistical results, interpersonal coordination and the coupled oscillator model works differently. The simulated series present very close dynamics, resulting from the continuous coupling of positions and velocities in the model. In contrast, experimental series appear more independent, at least on this local scale. In order to quantify these local dependences, we computed the average local cross-correlation coefficient, using a sliding window of 15 points (Marmelat and Delignieres 2012). The average crosscorrelation coefficient was 0.87 for simulated series, but close to zero for experimental series.

One could argue, however, that although the stiffness series $\omega_{1, i}$ and $\omega_{2, i}$ included in the model are independent, they still have the same average values, and that more realistic results could be obtained by introducing an asymmetry, i.e. a difference between the natural frequencies of the two systems. In order to check this point, we performed additional simulations introducing a 10\% detuning between the two oscillators (mean $\omega_{1, i}=4 \pi$, and mean $\omega_{2, i}=4.4 \pi$ ). All others parameters were unchanged. This new set of simulations gave essentially similar results, suggesting that the symmetry of the first model cannot per se explain the strong local convergence of the simulated series. We present in Figure 6 (panel e) an example subset of series simulated with these new settings. 

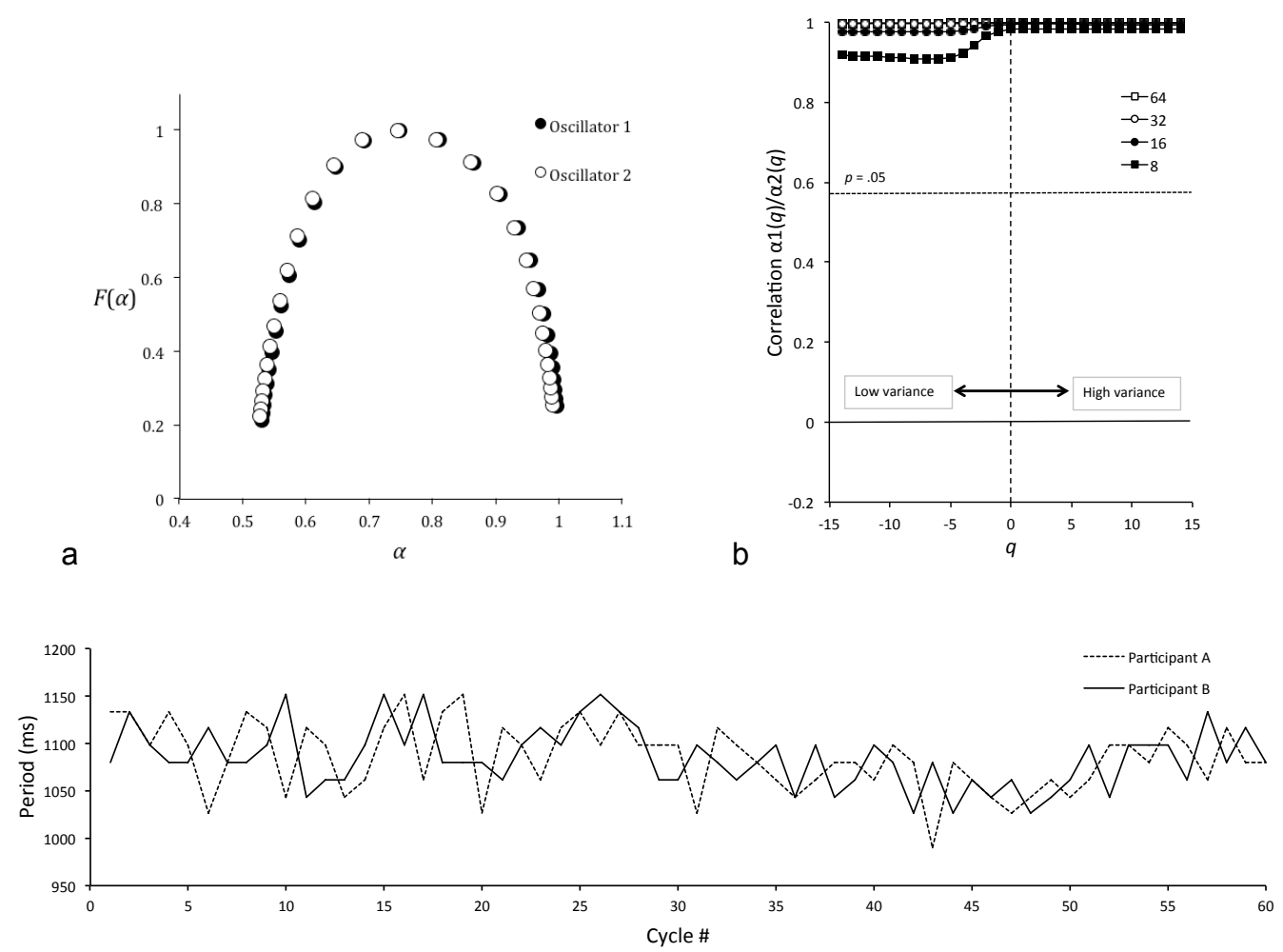

C

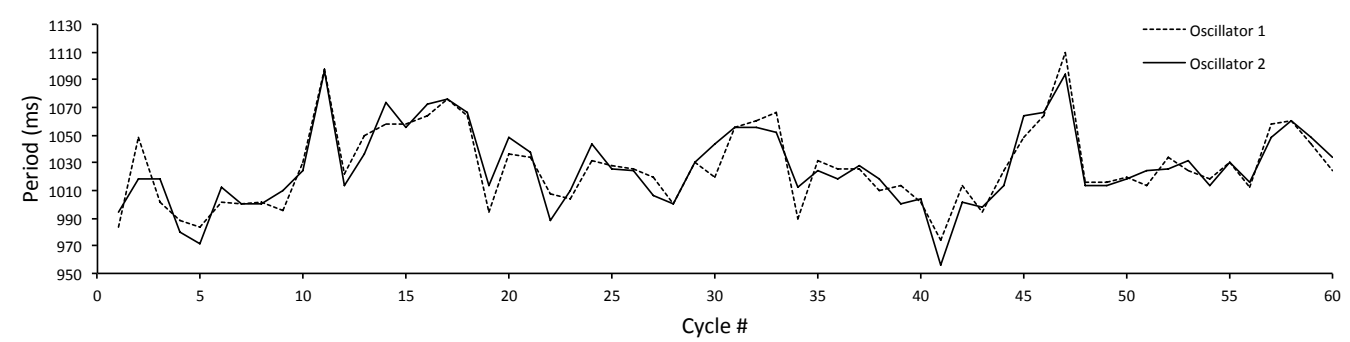

d

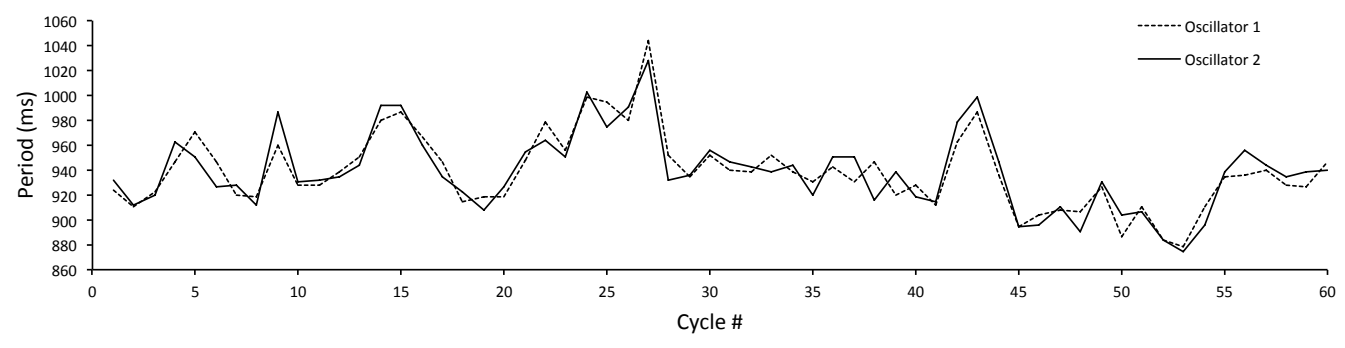

e

Figure 6: Panel a: Average multifractal spectra for the two simulated oscillators. Panel b: Correlation functions $\mathrm{r}(q)$, for the four ranges of intervals considered ( 8 to $N / 2,16$ to $N / 2,32$ to $N / 2$, and 64 to $N / 2$ ). Panel c: Representative subsets of coupled experimental series (50 points). Panel d: Representative subsets of coupled simulated series (symmetric model). Panel d: Representative subsets of coupled simulated series, with a $10 \%$ detuning.

These results suggest that a local continuous coupling, as proposed in the HKB model, can indeed mimic the statistical matching supposed to emerge from complexity matching. However, this kind of coupling generates a very close correspondence between the trajectories of oscillators, which seems unrealistic in view of the experimental observations. 


\section{Conclusion}

Complexity matching is a very innovative hypothesis, which has recently motivated a number of theoretical and experimental contributions. In this paper we introduce a method, based on multifractal analysis, for distinguishing between genuine complexity matching and local discrete coupling. Our results show that some situations that were considered prototypic of complexity matching, where participants had to synchronize with a fractal metronome, seem controlled through local adjustments. In contrast, genuine complexity matching seems occurring when two complex systems are mutually coupled.

Complexity matching and local discrete coupling, however, are not necessarily exclusive. One could conceive, for example, that in some tasks synchronization could be achieved through a mix of the two processes. Moreover, the respective contribution of each processes could differ among participants (see, for example, Delignieres and Torre 2011). Further investigations, focusing on individual series and based on crosscorrelations should provide some insights about this hypothesis.

\section{Conflict of interest}

The authors declare that they have no conflict of interest.

\section{Acknowledgments}

We thank Prof. Andras Eke who kindly provided us with the Matlab code for the multifractal focus-based method .

\section{References}

Abney DH, Paxton A, Dale R, Kello CT (2014) Complexity Matching in Dyadic Conversation. J Exp Psychol-Gen 143:2304-2315. doi: 10.1037/xge0000021

Almurad ZMH, Delignières D (2016) Evenly spacing in Detrended Fluctuation Analysis. Phys Stat Mech Its Appl 451:63-69. doi: 10.1016/j.physa.2015.12.155

Aquino G, Bologna M, Grigolini P, West BJ (2010) Beyond the Death of Linear Response: 1/f Optimal Information Transport. Phys Rev Lett 105:69901. doi: 10.1103/PhysRevLett.105.069901

Aquino G, Bologna M, West BJ, Grigolini P (2011) Transmission of information between complex systems: 1/f resonance. Phys Rev E 83:51130. doi:

10.1103/PhysRevE.83.051130

Assisi CG, Jirsa VK, Kelso JAS (2005) Dynamics of multifrequency coordination using parametric driving: theory and experiment. Biol Cybern 93:6-21. doi: 10.1007/s00422005-0558-y

Chen YQ, Ding MZ, Kelso JAS (1997) Long memory processes (1/f(alpha) type) in human coordination. Phys Rev Lett 79:4501-4504. doi: 10.1103/PhysRevLett.79.4501

Chen YQ, Ding MZ, Kelso JAS (2001) Origins of timing errors in human sensorimotor coordination. J Mot Behav 33:3-8.

Delignières D, Marmelat V (2014) Strong anticipation and long-range cross-correlation: Application of detrended cross-correlation analysis to human behavioral data. Phys Stat Mech Its Appl 394:47-60. 
Delignières D, Ramdani S, Lemoine L, et al (2006) Fractal analyses for "short" time series: A re-assessment of classical methods. J Math Psychol 50:525-544. doi: 10.1016/j.jmp.2006.07.004

Delignieres D, Torre K (2011) Event-Based and Emergent Timing: Dichotomy or Continuum? A Reply to Repp and Steinman (2010). J Mot Behav 43:311-318. doi: 10.1080/00222895.2011.588274

Delignières D, Torre K, Lemoine L (2008) Fractal models for event-based and dynamical timers. Acta Psychol (Amst) 127:382-397. doi: 10.1016/j.actpsy.2007.07.007

Delignières D, Torre K, Lemoine L (2005) Methodological issues in the application of monofractal analyses in psychological and behavioral research. Nonlinear Dyn Psychol Life Sci 9:435-61.

Fine JM, Likens AD, Amazeen EL, Amazeen PG (2015) Emergent Complexity Matching in Interpersonal Coordination: Local Dynamics and Global Variability. J Exp Psychol Hum Percept Perform. doi: 10.1037/xhp0000046

Fink PW, Foo P, Jirsa VK, Kelso JAS (2000) Local and global stabilization of coordination by sensory information. Exp Brain Res 134:9-20. doi: 10.1007/s002210000439

Gilden DL (1997) Fluctuations in the time required for elementary decisions. Psychol Sci 8:296-301. doi: 10.1111/j.1467-9280.1997.tb00441.x

Gilden DL (2001) Cognitive emissions of 1/f noise. Psychol Rev 108:33-56. doi: 10.1037/0033-295X.108.1.33

Haken H, Kelso J, Bunz H (1985) A Theoretical-Model of Phase-Transitions in Human Hand Movements. Biol Cybern 51:347-356. doi: 10.1007/BF00336922

Hove MJ, Suzuki K, Uchitomi H, et al (2012) Interactive Rhythmic Auditory Stimulation Reinstates Natural 1/f Timing in Gait of Parkinson's Patients. PloS One 7:e32600.

Ihlen EAF, Vereijken B (2010) Interaction-Dominant Dynamics in Human Cognition: Beyond 1/f(alpha) Fluctuation. J Exp Psychol-Gen 139:436-463. doi: $10.1037 / \mathrm{a} 0019098$

Kaipust JP, McGrath D, Mukherjee M, Stergiou N (2013) Gait variability is altered in older adults when listening to auditory stimuli with differing temporal structures. Ann Biomed Eng 41:1595-1603. doi: 10.1007/s10439-012-0654-9

Kantelhardt JW, Zschiegner SA, Koscielny-Bunde E, et al (2002) Multifractal detrended fluctuation analysis of nonstationary time series. Phys -Stat Mech Its Appl 316:87-114. doi: 10.1016/S0378-4371(02)01383-3

Leise T, Cohen A (2007) Nonlinear oscillators at our fingertips. Am Math Mon 114:14-28.

Mafahim JU, Lambert D, Zare M, Grigolini P (2015) Complexity matching in neural networks. New J Phys 17:15003. doi: 10.1088/1367-2630/17/1/015003

Makowiec D, Rynkiewicz A, Galaska R, et al (2011) Reading multifractal spectra: Aging by multifractal analysis of heart rate. Epl 94:68005. doi: 10.1209/0295-5075/94/68005 
Marmelat V, Delignieres D (2012) Strong anticipation: complexity matching in interpersonal coordination. Exp Brain Res 222:137-148. doi: 10.1007/s00221-0123202-9

Marmelat V, Delignières D (2011) Complexity, Coordination, and Health: Avoiding Pitfalls and Erroneous Interpretations in Fractal Analyses. Med-Lith 47:393-398.

Marmelat V, Torre K, Beek PJ, Daffertshofer A (2014) Persistent Fluctuations in Stride Intervals under Fractal Auditory Stimulation. Plos One 9:e91949. doi: 10.1371/journal.pone.0091949

Mukli P, Nagy Z, Eke A (2015) Multifractal formalism by enforcing the universal behavior of scaling functions. Phys Stat Mech Its Appl 417:150-167. doi: 10.1016/j.physa.2014.09.002

Oswiecimka P, Kwapien J, Drozdz S (2006) Wavelet versus detrended fluctuation analysis of multifractal structures. Phys Rev E 74:16103. doi:

10.1103/PhysRevE.74.016103

Peng CK, Mietus J, Hausdorff JM, et al (1993) Long-range anticorrelations and nonGaussian behavior of the heartbeat. Phys Rev Lett 70:1343-1346.

Podobnik B, Stanley HE (2008) Detrended cross-correlation analysis: A new method for analyzing two nonstationary time series. Phys Rev Lett 100:84102. doi:

10.1103/PhysRevLett.100.084102

Rhea CK, Kiefer AW, Wittstein MW, et al (2014) Fractal gait patterns are retained after entrainment to a fractal stimulus. PloS One 9:e106755. doi:

10.1371/journal.pone.0106755

Schöner G, Haken H, Kelso J (1986) A Stochastic-Theory of Phase-Transitions in Human Hand Movement. Biol Cybern 53:247-257. doi: 10.1007/BF00336995

Schumann AY, Kantelhardt JW (2011) Multifractal moving average analysis and test of multifractal model with tuned correlations. Phys -Stat Mech Its Appl 390:2637-2654. doi: 10.1016/j.physa.2011.03.002

Stephen DG, Dixon JA (2011) Strong anticipation: Multifractal cascade dynamics modulate scaling in synchronization behaviors. Chaos Solitons Fractals 44:160-168. doi: 10.1016/j.chaos.2011.01.005

Stephen DG, Stepp N, Dixon JA, Turvey MT (2008) Strong anticipation: Sensitivity to long-range correlations in synchronization behavior. Phys Stat Mech Its Appl 387:52715278.

Torre K, Delignières D (2008a) Distinct ways of timing movements in bimanual coordination tasks: Contribution of serial correlation analysis and implications for modeling. Acta Psychol (Amst) 129:284-296.

Torre K, Delignières D (2008b) Unraveling the finding of $1 / f($ beta) noise in self-paced and synchronized tapping: a unifying mechanistic model. Biol Cybern 99:159-170. doi: $10.1007 / \mathrm{s} 00422-008-0247-8$ 
Torre K, Delignières D (2009) Fractal dynamics of human gait: a reassessment of the 1996 data of Hausdorff et al. J Appl Physiol 106:1272-1279. doi:

10.1152/japplphysiol.90757.2008

Torre K, Varlet M, Marmelat V (2013) Predicting the biological variability of environmental rhythms: Weak or strong anticipation for sensorimotor synchronization? Brain Cogn 83:342-350. doi: 10.1016/j.bandc.2013.10.002

Torre K, Wagenmakers E-J (2009) Theories and models for 1/f(beta) noise in human movement science. Hum Mov Sci 28:297-318. doi: 10.1016/j.humov.2009.01.001

West BJ, Geneston EL, Grigolini P (2008) Maximizing information exchange between complex networks. Phys Rep-Rev Sect Phys Lett 468:1-99. doi:

10.1016/j.physrep.2008.06.003 